\title{
RELAXATION TO ISENTROPIC GAS DYNAMICS FOR A BGK SYSTEM WITH SINGLE KINETIC ENTROPY *
}

\author{
F. BERTHELIN ${ }^{\dagger}$ AND F. BOUCHUT ${ }^{\ddagger}$
}

\begin{abstract}
We introduce a family of vector kinetic BGK equations leading to isentropic gas dynamics in the relaxation limit, that have only one entropy at the kinetic level. These models possess the generic structure of kinetic relaxation models. By a sharp adaptation of averaging lemmas to BGK models that have a dissipative entropy, we establish an estimate in the inverse of the square root of the relaxation parameter on the $L^{2}$ norm of the gradient of the approximations. This estimation is new in the context of kinetic equations, and it allows, by the method of DiPerna, to establish the convergence towards weak solutions of isentropic gas dynamics that satisfy a single entropy inequality.
\end{abstract}

1. Introduction. The method developed by DiPerna [12] using Tartar's compensated compactness [25] for establishing the convergence of approximate solutions to one-dimensional nonlinear hyperbolic systems of conservation laws is well-known. It has been used mainly when dealing with viscosity or numerical approximations. However, the problem of establishing similar results to relaxation models such as those introduced in [11] is not solved in general. Apart from the scalar case, for which we refer to the review [27], until now, only particular relaxation structures have been treated in [26], [24], [3]. In [24] and [3], the main ingredient that is used is that many entropies have a kinetic extension, in the sense defined in [11]. A general method for finding such extensions in kinetic BGK models can be found in [6]. In physically relevant models however, only one entropy has an extension, and therefore one has to introduce other tools. This was done in [26], where from particular energy inequalities, the author establishes an estimate on the gradient of the approximate solutions

$$
\left\|\partial_{x} U_{\varepsilon}\right\|_{L_{t, x}^{2}} \leq \frac{C}{\sqrt{\varepsilon}}
$$

where $\varepsilon$ is the relaxation parameter. This is indeed exactly the estimate used by DiPerna in the viscosity approximation.

The purpose of this paper is to explain how this approach can indeed be used generically when dealing with kinetic BGK models, even if we have a single entropy extension. The new ingredient for establishing (1.1) is an averaging lemma which is particularly adapted to the BGK relaxation term. With different scalings, related appropriate sharp estimates for relaxation limits leading to incompressible models of gas dynamics in multidimension have recently been the object of great progress, see [10], [1], [19], [23], [15].

In order to be more specific, we shall deal with the one-dimensional system of isentropic gas dynamics

$$
\left\{\begin{array}{l}
\partial_{t} \rho+\partial_{x}(\rho u)=0, \\
\left.\partial_{t}(\rho u)+\partial_{x}\left(\rho u^{2}+\kappa \rho^{\gamma}\right)=0, \quad \text { in }\right] 0, \infty\left[{ }_{t} \times \mathbb{R}_{x}\right.
\end{array}\right.
$$

\footnotetext{
*Received May 3, 2002; accepted for publication November 4, 2002.

†Université d'Orléans, UMR 6628, Département de Mathématiques, BP 6759, 45067 Orléans cedex 2, France (Florent.Berthelin@labomath.univ-orleans.fr).

¥ Département de Mathématiques et Applications, Ecole Normale Supérieure et CNRS, UMR 8553, 45, rue d'Ulm, 75230 Paris cedex 05, France (Francois.Bouchut@ens.fr).
} 
with $\rho(t, x) \geq 0, u(t, x) \in \mathbb{R}$ and $\kappa>0,1<\gamma<3$. Approximate solutions are obtained via a kinetic BGK model

$$
\left.\partial_{t} f_{\varepsilon}+\xi \partial_{x} f_{\varepsilon}=\frac{M\left(\rho_{\varepsilon}, u_{\varepsilon}, \xi\right)-f_{\varepsilon}}{\varepsilon} \quad \text { in }\right] 0, \infty\left[{ }_{t} \times \mathbb{R}_{x} \times \mathbb{R}_{\xi},\right.
$$

with

$$
\left(\rho_{\varepsilon}, \rho_{\varepsilon} u_{\varepsilon}\right)=U_{\varepsilon}=\int f_{\varepsilon} d \xi
$$

Here $f_{\varepsilon}(t, x, \xi) \in \mathbb{R}^{2}$ as in the general framework of [6], and the maxwellian equilibrium $M$ has to satisfy the consistency relations

$$
\int M(\rho, u, \xi) d \xi=(\rho, \rho u), \quad \int \xi M(\rho, u, \xi) d \xi=F \equiv\left(\rho u, \rho u^{2}+\kappa \rho^{\gamma}\right) .
$$

The stability theory for (1.2), that has been completed in [21], [20], uses the entropy inequalities

$$
\partial_{t} \eta(U)+\partial_{x} G(U) \leq 0,
$$

for a whole family of couples entropy-entropy fluxes $(\eta, G)$, with $\eta$ convex, the so called weak entropies. According to [6], a kinetic entropy associated to $\eta$ is a function $H_{\eta}(f, \xi)$ which is convex in $f$, verifies the consistency relation

$$
\int H_{\eta}(M(\rho, u, \xi), \xi) d \xi=\eta(\rho, u)
$$

and satisfies a minimization principle

$$
\int H_{\eta}(M(\rho, u, \xi), \xi) d \xi \leq \int H_{\eta}(f(\xi), \xi) d \xi, \quad(\rho, \rho u)=\int f(\xi) d \xi
$$

for any function $f(\xi) \in L_{\xi}^{1}$ taking values in suitable convex sets $D_{\xi}$, in the sense that $f(\xi) \in D_{\xi}$. The model used in [2], [3] (see also [4] and [5] for related problems) possesses kinetic entropies $H_{\eta}$ for all entropies $\eta$. Here we shall consider more general models that have a single kinetic entropy $H$ corresponding to the physical energy,

$$
\eta^{e}(\rho, u)=\rho u^{2} / 2+\frac{\kappa}{\gamma-1} \rho^{\gamma}, \quad G^{e}(\rho, u)=\left(\eta^{e}(\rho, u)+\kappa \rho^{\gamma}\right) u .
$$

Therefore, we shall obtain in the relaxation limit weak solutions $U=(\rho, \rho u)$ satisfying only

$$
\partial_{t} \eta(U)+\partial_{x} G(U)=m_{\eta} \in \mathcal{M}_{l o c}
$$

for any (weak) entropy $\eta$, and the measure $m_{\eta}$ is nonpositive only for $\eta=\eta^{e}$. This is indeed the approach of [26], [17], [16]. Thus we look for approximate entropy equations

$$
\partial_{t} \eta\left(U_{\varepsilon}\right)+\partial_{x} G\left(U_{\varepsilon}\right)=m_{\eta, \varepsilon}+R_{\eta, \varepsilon}
$$

where $R_{\eta, \varepsilon} \rightarrow 0$ as $\varepsilon \rightarrow 0$ in $W_{l o c}^{-1, p}$ for any $1<p<\infty, m_{\eta, \varepsilon}$ are measures locally bounded uniformly in $\varepsilon$, and $m_{\eta^{e}, \varepsilon} \leq 0$. This is sufficient to apply the compensated compactness method. The measures $m_{\eta, \varepsilon}$ are indeed bounded via the estimate (1.1), 
as in the viscosity approximation. The fact that the energy has a kinetic extension comes into play only via an a priori estimate coming from the entropy dissipation,

$$
\left\|M_{\varepsilon}-f_{\varepsilon}\right\|_{L_{t x \xi}^{2}} \leq C \sqrt{\varepsilon}
$$

which enables to establish (1.1). However, to get this we need coercivity of the kinetic entropy, and this holds in our model only for $1<\gamma \leq 5 / 3$. We shall assume also some a priori $L^{\infty}$ bound on $\rho_{\varepsilon}, u_{\varepsilon}$ and that the vacuum does not appear. These are the main restrictions of our work. The convergence result can be stated as follows.

THEOREM 1.1. We assume that $1<\gamma \leq 5 / 3$, and that the solution $f_{\varepsilon}$ to (2.1)(2.10) with initial data $f_{\varepsilon}^{0}$ such that (2.22)-(2.24) and (4.2) hold, satisfies (2.35)(2.36) and (4.1). We set $U_{\varepsilon}=\left(\rho_{\varepsilon}, \rho_{\varepsilon} u_{\varepsilon}\right)=\int_{\mathbb{R}} f_{\varepsilon} d \xi$. Then there exists a subsequence such that $\rho_{\varepsilon} \rightarrow \rho$ and $\rho_{\varepsilon} u_{\varepsilon} \rightarrow \rho u$ a.e., where $(\rho, \rho u)$ is a weak solution to (1.2) with initial data $\left(\rho^{0}, \rho^{0} u^{0}\right)=w$ - $\lim \int f_{\varepsilon}^{0} d \xi$, and (1.10) holds.

The outline of the paper is as follows. In Section 2, we introduce our BGK model and establish the kinetic entropy dissipation estimate that leads to (1.12). In Section 3 , we prove the key averaging lemma. Finally, in Section 4 we establish the relaxation limit by combining the averaging lemma and the dissipation result, in order to obtain (1.1), (1.11), and Theorem 1.1.

2. Kinetic equation. In this section, we introduce a family of vector kinetic BGK models for isentropic gas dynamics, parametrized by a scalar $0<\theta \leq 1$. The value $\theta=(\gamma-1) / 2$ gives indeed the model of [2], [3], but for other values of $\theta$, only the energy of the system has a kinetic extension. This is the case in particular for the most physical model, obtained for $\theta=1$. We establish the estimates supplied by the corresponding dissipation in Theorem 2.5.

2.1. BGK model. The model we consider is written as

$$
\left.\partial_{t} f+\xi \partial_{x} f=\frac{M[f]-f}{\varepsilon} \quad \text { in }\right] 0, \infty[\times \mathbb{R} \times \mathbb{R}
$$

where $f=f(t, x, \xi)=\left(f_{0}(t, x, \xi), f_{1}(t, x, \xi)\right) \in \mathbb{R}^{2}, t \geq 0, x \in \mathbb{R}, \xi \in \mathbb{R}$,

$$
\begin{gathered}
f(t, x, \xi) \in D_{\xi}, \\
M[f](t, x, \xi)=M(\rho(t, x), u(t, x), \xi), \\
\rho(t, x)=\int_{\mathbb{R}} f_{0}(t, x, \xi) d \xi, \rho(t, x) u(t, x)=\int_{\mathbb{R}} f_{1}(t, x, \xi) d \xi,
\end{gathered}
$$

and

$$
\begin{gathered}
M(\rho, u, \xi)=\left(M_{0}(\rho, u, \xi), M_{1}(\rho, u, \xi)=((1-\theta) u+\theta \xi) M_{0}(\rho, u, \xi)\right) \\
M_{0}(\rho, u, \xi)=c_{\theta}\left(\frac{2 \gamma \kappa}{(\gamma-1) \theta} \rho^{\gamma-1}-(\xi-u)^{2}\right)_{+}^{\lambda} \\
\lambda=\frac{1}{\gamma-1}-\frac{1}{2}, c_{\theta}=\frac{1}{J_{\lambda}}\left(\frac{2 \gamma \kappa}{(\gamma-1) \theta}\right)^{-1 /(\gamma-1)}
\end{gathered}
$$




$$
J_{\lambda}=\int_{-1}^{1}\left(1-z^{2}\right)^{\lambda} d z=\sqrt{\pi} \Gamma(\lambda+1) / \Gamma(\lambda+3 / 2) .
$$

The parameter $\theta$ must satisfy

$$
0<\theta \leq 1,
$$

and the model of [2], [3] corresponds to the choice $\theta=(\gamma-1) / 2$. The sets $D_{\xi}$ of $(2.2)$ are defined as

$$
\begin{aligned}
D_{\xi}=D & =\left\{\left(f_{0}, f_{1}\right) \in \mathbb{R}^{2}, f_{0}>0 \text { or } f_{1}=f_{0}=0\right\} \quad \text { if } \theta<1, \\
D_{\xi} & =\left\{\left(f_{0}, f_{1}\right) \in \mathbb{R}^{2}, f_{0} \geq 0, f_{1}=\xi f_{0}\right\} \quad \text { if } \theta=1 .
\end{aligned}
$$

The kinetic entropy for (2.1) is defined as follows. In the case $\theta<1$, for $f=\left(f_{0}, f_{1}\right) \in$ $D \backslash\{(0,0)\}$,

$$
\begin{aligned}
H(f, \xi) & =\frac{\theta}{1-\theta} \frac{\xi^{2}}{2} f_{0}+\frac{\theta}{2 c_{\theta}^{1 / \lambda}} \frac{f_{0}^{1+1 / \lambda}}{1+1 / \lambda}+\frac{1}{1-\theta} \frac{1}{2} \frac{f_{1}^{2}}{f_{0}}-\frac{\theta}{1-\theta} \xi f_{1} \\
& =\frac{1}{1-\theta} \frac{1}{2}\left(\frac{f_{1}}{\sqrt{f_{0}}}-\theta \xi \sqrt{f_{0}}\right)^{2}+\frac{\theta}{2 c_{\theta}^{1 / \lambda}} \frac{f_{0}^{1+1 / \lambda}}{1+1 / \lambda}+\theta \frac{\xi^{2}}{2} f_{0} \geq 0
\end{aligned}
$$

and $H(0, \xi)=0$, while in the case $\theta=1$, for $f \in D_{\xi}$,

$$
H(f, \xi)=\frac{\xi^{2}}{2} f_{0}+\frac{1}{2 c_{1}^{1 / \lambda}} \frac{f_{0}^{1+1 / \lambda}}{1+1 / \lambda} .
$$

Indeed, making $f_{1}=\xi f_{0}$ in (2.11) and letting $\theta \rightarrow 1$ gives (2.12).

The value of the moments of $M$ are

$$
\begin{gathered}
\int_{\mathbb{R}} M d \xi=(\rho, \rho u), \quad \int_{\mathbb{R}} \xi M d \xi=\left(\rho u, \rho u^{2}+\kappa \rho^{\gamma}\right), \\
\int_{\mathbb{R}} \xi^{2} M d \xi=\left(\rho u^{2}+\frac{\kappa \rho^{\gamma}}{\theta},\left(\rho u^{2}+\left(2+\frac{1}{\theta}\right) \kappa \rho^{\gamma}\right) u\right),
\end{gathered}
$$

for every $\rho \geq 0$ and $u \in \mathbb{R}$. These relations enable to compute the formal ChapmanEnskog expansion for this model, which is, up to terms in $\varepsilon^{2}$,

$$
\partial_{t} U+\partial_{x} F(U)=\varepsilon \partial_{x}\left(D(U) \partial_{x} U\right),
$$

with

$$
D(U)=\left(\begin{array}{cc}
(1 / \theta-1) \gamma \kappa \rho^{\gamma-1} & 0 \\
((\gamma-1) / \theta-2) \kappa \rho^{\gamma-1} u & (2-\gamma+1 / \theta) \kappa \rho^{\gamma-1}
\end{array}\right),
$$

and $U=(\rho, \rho u)$. We observe that the least dissipative model is obtained for $\theta=1$, where $D(U)$ becomes singular. This model is degenerate also because $M(\rho, u, \xi)$ is then colinear to $(1, \xi)$, thus we have to look for solutions $f=\left(f_{0}, \xi f_{0}\right)$. This is the most physically relevant model, that is already mentioned in [6]. Our analysis also works in this case. 
We have the following relations, that can be obtained with straightforward computations.

Proposition 2.1. The energy $\eta^{e}$ and the kinetic entropy $H$ are related through

$$
\begin{gathered}
\int_{\mathbb{R}} H(M(\rho, u, \xi), \xi) d \xi=\eta^{e}(\rho, u) \quad \text { for every } \rho \geq 0, u \in \mathbb{R}, \\
\int_{\mathbb{R}} \xi H(M(\rho, u, \xi), \xi) d \xi=G^{e}(\rho, u) \quad \text { for every } \rho \geq 0, u \in \mathbb{R},
\end{gathered}
$$

and if $\theta<1$,

$$
\left\{\begin{array}{l}
\frac{\partial H}{\partial f_{0}}(M(\rho, u, \xi), \xi)=\frac{\partial \eta^{e}}{\partial \rho}(\rho, u)=\frac{\gamma}{\gamma-1} \kappa \rho^{\gamma-1}-u^{2} / 2 \\
\frac{\partial H}{\partial f_{1}}(M(\rho, u, \xi), \xi)=\frac{\partial \eta^{e}}{\partial(\rho u)}(\rho, u)=u
\end{array}\right.
$$

for every $\rho>0, u \in \mathbb{R}$ and $\xi \in \mathbb{R}$ such that $M_{0}(\rho, u, \xi)>0$.

With the same proofs as in [2], one can deduce the following results.

Proposition 2.2 (Subdifferential inequality). For any $\rho \geq 0, u, \xi \in \mathbb{R}$ and $f \in D_{\xi}$, we have

$$
H(f, \xi) \geq H(M(\rho, u, \xi), \xi)+\left(\frac{\gamma \kappa}{\gamma-1} \rho^{\gamma-1}-\frac{u^{2}}{2}, u\right) \cdot(f-M(\rho, u, \xi)) .
$$

Proposition 2.3 (Minimization principle). Consider $f \in L^{1}\left(\mathbb{R}_{\xi}\right)$ such that $f(\xi) \in D_{\xi}$ a.e. and $\int_{\mathbb{R}} H(f(\xi), \xi) d \xi<\infty$. Then

$$
\int_{\mathbb{R}} H(M[f](\xi), \xi) d \xi \leq \int_{\mathbb{R}} H(f(\xi), \xi) d \xi .
$$

2.2. Dissipation estimates. We take an initial data $f_{\varepsilon}^{0} \in L^{1}\left(\mathbb{R}_{x} \times \mathbb{R}_{\xi}\right)$ which satisfies

$$
\begin{gathered}
f_{\varepsilon}^{0}(x, \xi) \in D_{\xi} \text { a.e. in } \mathbb{R} \times \mathbb{R}, \\
\iint_{\mathbb{R} \times \mathbb{R}}\left|f_{\varepsilon}^{0}(x, \xi)\right| d x d \xi \leq C_{I}^{0}<\infty, \\
\iint_{\mathbb{R} \times \mathbb{R}} H\left(f_{\varepsilon}^{0}(x, \xi), \xi\right) d x d \xi \leq C_{H}^{0}<\infty .
\end{gathered}
$$

Then with the same proof as in [2], there exists a solution $f_{\varepsilon}$ to the BGK model (2.1)-(2.10), with initial data $f_{\varepsilon}^{0}$, satisfying

$$
f_{\varepsilon} \in C_{t} \cap L_{t}^{\infty}\left(\left[0, \infty\left[, L^{1}\left(\mathbb{R}_{x} \times \mathbb{R}_{\xi}\right)\right),\right.\right.
$$




$$
\begin{gathered}
\forall t \geq 0, \quad \iint_{\mathbb{R} \times \mathbb{R}} f_{\varepsilon}(t, x, \xi) d x d \xi=\iint_{\mathbb{R} \times \mathbb{R}} f_{\varepsilon}^{0}(x, \xi) d x d \xi, \\
\forall t \geq 0, \quad \iint_{\mathbb{R} \times \mathbb{R}} H\left(f_{\varepsilon}(t, x, \xi), \xi\right) d x d \xi \leq C_{H}^{0}, \\
\partial_{t} \int f_{\varepsilon} d \xi+\partial_{x} \int \xi f_{\varepsilon} d \xi=0 .
\end{gathered}
$$

As in [2], we have the following identity.

Proposition 2.4 (Entropy variation). The solution $f_{\varepsilon}$ to (2.1)-(2.10), with initial data $f_{\varepsilon}^{0}$ satisfying (2.22)-(2.24), verifies

$$
\begin{aligned}
& {\left[\iint_{\mathbb{R} \times \mathbb{R}} H\left(f_{\varepsilon}(t, x, \xi), \xi\right) d x d \xi\right]_{0}^{T} } \\
= & \frac{1}{\varepsilon} \iiint_{0, T[\times \mathbb{R} \times \mathbb{R}}\left(H^{\prime}\left(f_{\varepsilon}, \xi\right)-\left(\frac{\gamma \kappa}{\gamma-1} \rho_{\varepsilon}^{\gamma-1}-\frac{u_{\varepsilon}^{2}}{2}, u_{\varepsilon}\right)\right) \cdot\left(M\left[f_{\varepsilon}\right]-f_{\varepsilon}\right) d t d x d \xi .
\end{aligned}
$$

Moreover,

$$
\left(H^{\prime}\left(f_{\varepsilon}, \xi\right)-\left(\frac{\gamma \kappa}{\gamma-1} \rho_{\varepsilon}^{\gamma-1}-\frac{u_{\varepsilon}^{2}}{2}, u_{\varepsilon}\right)\right) \cdot\left(M\left[f_{\varepsilon}\right]-f_{\varepsilon}\right) \leq 0 \quad \text { a.e. }
$$

In the case $\theta<1$, the derivative $H^{\prime}(f, \xi)$ of $H(f, \xi)$ with respect to $f$ is given for $f \neq 0$ by

$$
H^{\prime}(f, \xi)=\left(\frac{\theta}{1-\theta} \frac{\xi^{2}}{2}+\frac{\theta}{2 c_{\theta}^{1 / \lambda}} f_{0}^{1 / \lambda}-\frac{1}{1-\theta} \frac{1}{2} \frac{f_{1}^{2}}{f_{0}^{2}},-\frac{\theta}{1-\theta} \xi+\frac{1}{1-\theta} \frac{f_{1}}{f_{0}}\right),
$$

while if $\theta=1$, we set by convention

$$
H^{\prime}(f, \xi)=\left(\frac{\xi^{2}}{2}+\frac{1}{2 c_{1}^{1 / \lambda}} f_{0}^{1 / \lambda}, 0\right) .
$$

In $(2.30), H^{\prime}(0, \xi)$ needs not be defined since by applying the result of [7] to each component of $f_{\varepsilon}$, we have

$$
M\left[f_{\varepsilon}\right]-f_{\varepsilon}=0 \quad \text { a.e. } t, x, \xi \text { where } f_{\varepsilon}=0 .
$$

We shall now assume $L^{\infty}$ bounds, because we are not able to prove them. It was possible in [3] because of the compatibility with all entropies, but here this property does not hold. Thus we assume that for some $F, A>0$ independent of $\varepsilon$,

$$
\left|f_{\varepsilon}\right|,\left|M\left[f_{\varepsilon}\right]\right| \leq F, \quad\left|\left(f_{\varepsilon}\right)_{1}\right| \leq A\left(f_{\varepsilon}\right)_{0},\left|\left(M\left[f_{\varepsilon}\right]\right)_{1}\right| \leq A\left(M\left[f_{\varepsilon}\right]\right)_{0} .
$$


We assume also compact support in velocity, that is to say there exists a closed ball $\bar{B}(0, Q)$ such that

$$
\operatorname{supp}_{\xi} f_{\varepsilon}, \operatorname{supp}_{\xi} M\left[f_{\varepsilon}\right] \subset \bar{B}(0, Q) .
$$

These assumptions imply obviously that $\rho_{\varepsilon}, u_{\varepsilon}$ are bounded independently of $\varepsilon$. We are now able to establish the optimal rate of convergence for $M\left[f_{\varepsilon}\right]-f_{\varepsilon}$.

TheOREm 2.5. We assume that $\lambda \geq 1$, that is to say $1<\gamma \leq 5 / 3$, and that the solution $f_{\varepsilon}$ to (2.1)-(2.10) with initial data $f_{\varepsilon}^{0}$ such that (2.22)-(2.24) hold, satisfies (2.35). Then there exists $C>0$ independent of $\varepsilon$, such that

$$
\iiint_{] 0, \infty[\times \mathbb{R} \times \mathbb{R}}\left|f_{\varepsilon}-M\left[f_{\varepsilon}\right]\right|^{2} d t d x d \xi \leq C \varepsilon .
$$

Proof. Since the case $\theta=1$ is the easiest and is performed with the same type of estimates, we let it to the reader, and consider only the case $\theta<1$. We start from the dissipation identity of Proposition 2.4,

$$
\iiint_{] 0, \infty[\times \mathbb{R} \times \mathbb{R}}\left(H^{\prime}\left(f_{\varepsilon}, \xi\right)-\left(\frac{\gamma \kappa}{\gamma-1} \rho_{\varepsilon}^{\gamma-1}-\frac{u_{\varepsilon}^{2}}{2}, u_{\varepsilon}\right)\right) \cdot\left(f_{\varepsilon}-M\left[f_{\varepsilon}\right]\right) d t d x d \xi \leq C_{H}^{0} \varepsilon .
$$

We note that whenever $M\left[f_{\varepsilon}\right] \neq 0$,

$$
\left(\frac{\gamma \kappa}{\gamma-1} \rho_{\varepsilon}^{\gamma-1}-\frac{u_{\varepsilon}^{2}}{2}, u_{\varepsilon}\right)=H^{\prime}\left(M\left[f_{\varepsilon}\right], \xi\right) .
$$

We also notice that for $X=\left(X_{0}, X_{1}\right) \in D \backslash\{0\}, Y=\left(Y_{0}, Y_{1}\right) \in \mathbb{R}^{2}$,

$$
H^{\prime \prime}(X, \xi) \cdot Y \cdot Y=K_{1} X_{0}^{1 / \lambda-1} Y_{0}^{2}+K_{2}\left(X_{1} Y_{0}-X_{0} Y_{1}\right)^{2} / X_{0}^{3},
$$

with $K_{1}=\theta / 2 \lambda c_{\theta}^{1 / \lambda}$ and $K_{2}=1 /(1-\theta)$. We deduce as in [3] that

$$
\begin{aligned}
& \mathbf{I}_{M\left[f_{\varepsilon}\right] \neq 0}\left(H^{\prime}\left(M\left[f_{\varepsilon}\right], \xi\right)-H^{\prime}\left(f_{\varepsilon}, \xi\right)\right) \cdot\left(M\left[f_{\varepsilon}\right]-f_{\varepsilon}\right) \\
\geq & K_{1} F^{1 / \lambda-1}\left(\left(M\left[f_{\varepsilon}\right]\right)_{0}-\left(f_{\varepsilon}\right)_{0}\right)^{2} \mathbf{I}_{M\left[f_{\varepsilon}\right] \neq 0},
\end{aligned}
$$

thus

$$
\iiint_{M\left[f_{\varepsilon}\right] \neq 0}\left(\left(f_{\varepsilon}\right)_{0}-\left(M\left[f_{\varepsilon}\right]\right)_{0}\right)^{2} d t d x d \xi \leq \frac{F^{1-1 / \lambda} C_{H}^{0}}{K_{1}} \varepsilon .
$$

We also have, recalling that by $(2.34), M\left[f_{\varepsilon}\right]=0$ a.e. where $f_{\varepsilon}=0$,

$$
\begin{aligned}
& \mathbf{I}_{M\left[f_{\varepsilon}\right] \neq 0}\left(H^{\prime}\left(M\left[f_{\varepsilon}\right], \xi\right)-H^{\prime}\left(f_{\varepsilon}, \xi\right)\right) \cdot\left(M\left[f_{\varepsilon}\right]-f_{\varepsilon}\right) \\
\geq & \frac{K_{2}}{F}\left(\frac{\left(f_{\varepsilon}\right)_{1}\left(M\left[f_{\varepsilon}\right]\right)_{0}-\left(f_{\varepsilon}\right)_{0}\left(M\left[f_{\varepsilon}\right]\right)_{1}}{\left(f_{\varepsilon}\right)_{0}+\left(M\left[f_{\varepsilon}\right]\right)_{0}}\right)^{2} \mathbf{I}_{M\left[f_{\varepsilon}\right] \neq 0} .
\end{aligned}
$$

In the case $\left(f_{\varepsilon}\right)_{0} \leq\left(M\left[f_{\varepsilon}\right]\right)_{0}$, we write

$$
\begin{aligned}
& \left(f_{\varepsilon}\right)_{1}-\left(M\left[f_{\varepsilon}\right]\right)_{1} \\
= & \frac{\left(f_{\varepsilon}\right)_{1}\left(M\left[f_{\varepsilon}\right]\right)_{0}-\left(f_{\varepsilon}\right)_{0}\left(M\left[f_{\varepsilon}\right]\right)_{1}}{\left(M\left[f_{\varepsilon}\right]\right)_{0}}-\left(\left(M\left[f_{\varepsilon}\right]\right)_{0}-\left(f_{\varepsilon}\right)_{0}\right) \frac{\left(M\left[f_{\varepsilon}\right]\right)_{1}}{\left(M\left[f_{\varepsilon}\right]\right)_{0}}
\end{aligned}
$$


and in the case $\left(f_{\varepsilon}\right)_{0}>\left(M\left[f_{\varepsilon}\right]\right)_{0}$, we write

$$
\begin{aligned}
& \left(f_{\varepsilon}\right)_{1}-\left(M\left[f_{\varepsilon}\right]\right)_{1} \\
= & \frac{\left(f_{\varepsilon}\right)_{1}\left(M\left[f_{\varepsilon}\right]\right)_{0}-\left(f_{\varepsilon}\right)_{0}\left(M\left[f_{\varepsilon}\right]\right)_{1}}{\left(f_{\varepsilon}\right)_{0}}-\left(\left(M\left[f_{\varepsilon}\right]\right)_{0}-\left(f_{\varepsilon}\right)_{0}\right) \frac{\left(f_{\varepsilon}\right)_{1}}{\left(f_{\varepsilon}\right)_{0}} .
\end{aligned}
$$

In any case, we obtain

$$
\begin{aligned}
& \mathbf{I}_{M\left[f_{\varepsilon}\right] \neq 0}\left(\left(f_{\varepsilon}\right)_{1}-\left(M\left[f_{\varepsilon}\right]\right)_{1}\right)^{2} \\
\leq & 8 \mathbf{I}_{M\left[f_{\varepsilon}\right] \neq 0}\left(\frac{\left(f_{\varepsilon}\right)_{1}\left(M\left[f_{\varepsilon}\right]\right)_{0}-\left(f_{\varepsilon}\right)_{0}\left(M\left[f_{\varepsilon}\right]\right)_{1}}{\left(f_{\varepsilon}\right)_{0}+\left(M\left[f_{\varepsilon}\right]\right)_{0}}\right)^{2} \\
& +2 A^{2} \mathbf{I}_{M\left[f_{\varepsilon}\right] \neq 0}\left(\left(M\left[f_{\varepsilon}\right]\right)_{0}-\left(f_{\varepsilon}\right)_{0}\right)^{2},
\end{aligned}
$$

thus we get

$$
\iiint_{M\left[f_{\varepsilon}\right] \neq 0}\left(\left(f_{\varepsilon}\right)_{1}-\left(M\left[f_{\varepsilon}\right]\right)_{1}\right)^{2} d t d x d \xi \leq\left(\frac{8 F C_{H}^{0}}{K_{2}}+\frac{2 A^{2} F^{1-1 / \lambda} C_{H}^{0}}{K_{1}}\right) \varepsilon .
$$

Let us now treat the set $\left\{M\left[f_{\varepsilon}\right]=0 ; f_{\varepsilon} \neq 0\right\}$. On this set, we have

$$
\begin{aligned}
& \left(H^{\prime}\left(f_{\varepsilon}, \xi\right)-\left(\frac{\gamma \kappa}{\gamma-1} \rho_{\varepsilon}^{\gamma-1}-\frac{u_{\varepsilon}^{2}}{2}, u_{\varepsilon}\right)\right) \cdot\left(f_{\varepsilon}-M\left[f_{\varepsilon}\right]\right) \\
= & {\left[\frac{\theta}{1-\theta} \frac{\xi^{2}}{2}+\frac{u_{\varepsilon}^{2}}{2}-\frac{\gamma \kappa}{\gamma-1} \rho_{\varepsilon}^{\gamma-1}\right]\left(f_{\varepsilon}\right)_{0}+\frac{\theta}{2 c_{\theta}^{1 / \lambda}}\left(f_{\varepsilon}\right)_{0}^{1+1 / \lambda} } \\
& +\frac{1}{1-\theta} \frac{1}{2} \frac{\left(f_{\varepsilon}\right)_{1}^{2}}{\left(f_{\varepsilon}\right)_{0}}-\left(\frac{\theta}{1-\theta} \xi+u_{\varepsilon}\right)\left(f_{\varepsilon}\right)_{1} \\
= & \frac{1}{2(1-\theta)}\left[\frac{\left(f_{\varepsilon}\right)_{1}}{\sqrt{\left(f_{\varepsilon}\right)_{0}}}-\left(\theta \xi+(1-\theta) u_{\varepsilon}\right) \sqrt{\left(f_{\varepsilon}\right)_{0}}\right]^{2}+\frac{\theta}{2 c_{\theta}^{1 / \lambda}}\left(f_{\varepsilon}\right)_{0}^{1+1 / \lambda} \\
& +\left[\frac{\theta}{2}\left(\xi-u_{\varepsilon}\right)^{2}-\frac{\gamma \kappa}{\gamma-1} \rho_{\varepsilon}^{\gamma-1}\right]\left(f_{\varepsilon}\right)_{0} .
\end{aligned}
$$

On the set $\left\{M\left[f_{\varepsilon}\right]=0 ; f_{\varepsilon} \neq 0\right\}$, we have $\rho_{\varepsilon}>0$, and $\frac{\theta}{2}\left(\xi-u_{\varepsilon}\right)^{2}-\frac{\gamma \kappa}{\gamma-1} \rho_{\varepsilon}^{\gamma-1}$ is nonnegative by definition of $M$, thus (2.48) and (2.38) give

$$
\iiint_{M\left[f_{\varepsilon}\right]=0}\left(f_{\varepsilon}\right)_{0}^{1+1 / \lambda} d t d x d \xi \leq \frac{2 c_{\theta}^{1 / \lambda} C_{H}^{0}}{\theta} \varepsilon .
$$

Now, since $\lambda \geq 1$, we have

$$
\begin{aligned}
\iiint_{M\left[f_{\varepsilon}\right]=0}\left(\left(f_{\varepsilon}\right)_{0}-\left(M\left[f_{\varepsilon}\right]\right)_{0}\right)^{2} d t d x d \xi & =\iiint_{M\left[f_{\varepsilon}\right]=0}\left(f_{\varepsilon}\right)_{0}^{2} d t d x d \xi \\
& \leq F^{1-1 / \lambda} \iiint_{M\left[f_{\varepsilon}\right]=0}\left(f_{\varepsilon}\right)_{0}^{1+1 / \lambda} d t d x d \xi \\
& \leq \frac{2 c_{\theta}^{1 / \lambda} C_{H}^{0} F^{1-1 / \lambda}}{\theta} \varepsilon,
\end{aligned}
$$


which gives the bound on the first component. Furthermore,

$$
\begin{aligned}
\mathbf{I}_{M\left[f_{\varepsilon}\right]=0}\left(\left(f_{\varepsilon}\right)_{1}-\left(M\left[f_{\varepsilon}\right]\right)_{1}\right)^{2} & =\mathbf{I}_{M\left[f_{\varepsilon}\right]=0}\left(f_{\varepsilon}\right)_{1}^{2} \\
& \leq A^{2} \mathbf{I}_{M\left[f_{\varepsilon}\right]=0}\left(f_{\varepsilon}\right)_{0}^{2} \\
& =A^{2} \mathbf{I}_{M\left[f_{\varepsilon}\right]=0}\left(\left(f_{\varepsilon}\right)_{0}-\left(M\left[f_{\varepsilon}\right]\right)_{0}\right)^{2},
\end{aligned}
$$

which concludes the proof.

3. Averaging. The regularity theory for averages of kinetic equations has been developed in [14], [13], [9], [22], [10], [18], [8]. We introduce here a result that is particularly adapted to BGK right-hand sides. In order to treat correctly the initial data, we use a Fourier transform in the variable $x$ only, in the spirit of [9]. The dual variable is denoted by $k$.

Proposition 3.1. Let $a \in L_{\text {loc }}^{\infty}\left(\mathbb{R}^{M}, \mathbb{R}^{N}\right)$ and $\psi \in L^{2}\left(\mathbb{R}^{M}\right)$, such that for some $K \geq 0$ and $0<\alpha \leq 1$, one has

$$
\forall \sigma \in S^{N-1}, \forall z \in \mathbb{R}, \forall \eta>0, \quad \int_{z<a(\xi) \cdot \sigma<z+\eta}|\psi(\xi)|^{2} d \xi \leq K \eta^{\alpha} .
$$

Let $f \in C\left([0, T], L^{2}\left(\mathbb{R}_{x}^{N} \times \mathbb{R}_{\xi}^{M}\right)\right)$ solve

$$
\left.\partial_{t} f+\operatorname{div}_{x}[a(\xi) f]=\frac{h-f}{\varepsilon}+g \quad \text { in }\right] 0, T\left[\times \mathbb{R}_{x}^{N} \times \mathbb{R}_{\xi}^{M},\right.
$$

for some $g, h \in L^{2}(] 0, T\left[\times \mathbb{R}_{x}^{N} \times \mathbb{R}_{\xi}^{M}\right)$ and initial data $f(0,)=.f^{0}$, and define

$$
\rho_{\psi}(t, x)=\int_{\mathbb{R}^{M}} f(t, x, \xi) \psi(\xi) d \xi \in C\left([0, T], L^{2}\left(\mathbb{R}_{x}^{N}\right)\right) .
$$

Then, for a.e. $k$,

$$
\begin{aligned}
& \left(\int_{0}^{T}\left|\widehat{\rho_{\psi}}(t, k)\right|^{2} d t\right)^{1 / 2} \\
\leq & \frac{C \sqrt{K}}{|k|^{\alpha / 2}}\left[\varepsilon^{\frac{1-\alpha}{2}}\left(\int\left|\widehat{f^{0}}\right|^{2} d \xi\right)^{1 / 2}+\left(\int\left|\widehat{f^{0}}\right|^{2} d \xi\right)^{\alpha / 2}\left(\int_{0}^{T} \int|\widehat{h}|^{2} d t d \xi\right)^{\frac{1}{2}(1-\alpha)}\right. \\
& +\varepsilon^{1-\frac{\alpha}{2}}\left(\int_{0}^{T} \int|\widehat{g}|^{2} d t d \xi\right)^{1 / 2}+\left(\int_{0}^{T} \int|\widehat{h}|^{2} d t d \xi\right)^{\frac{1}{2}\left(1-\frac{\alpha}{2}\right)}\left(\int_{0}^{T} \int|\widehat{g}|^{2} d t d \xi\right)^{\alpha / 4} \\
& \left.+\frac{1}{\varepsilon^{\alpha / 2}}\left(\int_{0}^{T} \int|\widehat{h}|^{2} d t d \xi\right)^{\frac{1}{2}\left(1-\frac{\alpha}{2}\right)}\left(\int_{0}^{T} \int|\widehat{h}-\widehat{f}|^{2} d t d \xi\right)^{\alpha / 4}\right]
\end{aligned}
$$

Proof. Denote $\underline{g}=\frac{h-f}{\varepsilon}+g \in L^{2}(] 0, T\left[\times \mathbb{R}_{x}^{N} \times \mathbb{R}_{\xi}^{M}\right)$. Then

$$
\left.\partial_{t} f+\operatorname{div}_{x}[a(\xi) f]=\underline{g} \quad \text { in }\right] 0, T\left[\times \mathbb{R}_{x}^{N} \times \mathbb{R}_{\xi}^{M},\right.
$$

and by estimate (3.23) in [9], the Fourier transform in $x, \widehat{\rho_{\psi}}(t, k)$ satisfies for a.e. $k$

$$
\begin{aligned}
\left(\int_{0}^{T}\left|\widehat{\rho_{\psi}}(t, k)\right|^{2} d t\right)^{1 / 2} \leq & \frac{C \sqrt{K}}{|k|^{\alpha / 2}}\left[\frac{1}{\lambda^{\frac{1-\alpha}{2}}}\left(\int\left|\widehat{f^{0}}(k, \xi)\right|^{2} d \xi\right)^{1 / 2}\right. \\
& \left.+\frac{1}{\lambda^{1-\frac{\alpha}{2}}}\left(\int_{0}^{T} \int|\lambda \widehat{f}+\widehat{g}|^{2}(t, k, \xi) d t d \xi\right)^{1 / 2}\right]
\end{aligned}
$$


for any function $\lambda=\lambda(k)>0$. Since

$$
\lambda \widehat{f}+\underline{\underline{g}}=\lambda \widehat{h}+\left(\frac{1}{\varepsilon}-\lambda\right)(\widehat{h}-\widehat{f})+\widehat{g}
$$

we deduce that

$$
\begin{aligned}
& \left(\int_{0}^{T}\left|\widehat{\rho_{\psi}}(t, k)\right|^{2} d t\right)^{1 / 2} \\
\leq & \frac{C \sqrt{K}}{|k|^{\alpha / 2}}\left[\frac{1}{\lambda^{\frac{1-\alpha}{2}}}\left(\int\left|\widehat{f^{0}}\right|^{2} d \xi\right)^{1 / 2}+\lambda^{\alpha / 2}\left(\int_{0}^{T} \int|\widehat{h}|^{2} d t d \xi\right)^{1 / 2}\right. \\
& \left.+\frac{1}{\lambda^{1-\frac{\alpha}{2}}}\left(\int_{0}^{T} \int|\widehat{g}|^{2} d t d \xi\right)^{1 / 2}+\frac{\left|\frac{1}{\varepsilon}-\lambda\right|}{\lambda^{1-\frac{\alpha}{2}}}\left(\int_{0}^{T} \int|\widehat{h}-\widehat{f}|^{2} d t d \xi\right)^{1 / 2}\right] .
\end{aligned}
$$

By taking

$$
\lambda=\min \left(\frac{1}{\varepsilon}, \frac{\frac{1}{\varepsilon}\left(\iint|\widehat{h}-\widehat{f}|^{2} d t d \xi\right)^{1 / 2}+\left(\iint|\widehat{g}|^{2} d t d \xi\right)^{1 / 2}}{\left(\iint|\widehat{h}|^{2} d t d \xi\right)^{1 / 2}}+\frac{\int\left|\widehat{f}^{0}\right|^{2} d \xi}{\iint \mid \widehat{h}^{2} d t d \xi}\right),
$$

we get the result.

We can notice that in the important case $\alpha=1$, the last term of the second argument in the right-hand side of (3.9) can be omitted.

Corollary 3.2. If in Proposition 3.1 we have $h \in H_{x}^{1}(] 0, T\left[\times \mathbb{R}_{x}^{N} \times \mathbb{R}_{\xi}^{M}\right)$ and $f^{0} \in$ $H_{x}^{1 / 2} \cap H_{x}^{1-\alpha / 2}\left(\mathbb{R}_{x}^{N} \times \mathbb{R}_{\xi}^{M}\right), g \in H_{x}^{1-\alpha / 2}(] 0, T\left[\times \mathbb{R}_{x}^{N} \times \mathbb{R}_{\xi}^{M}\right)$, then $\rho_{\psi} \in H_{x}^{1}(] 0, T\left[\times \mathbb{R}_{x}^{N}\right)$, with

$$
\begin{aligned}
\left\|\rho_{\psi}\right\|_{\dot{H}_{x}^{1}} \leq C_{N} \sqrt{K} & \left(\varepsilon^{\frac{1-\alpha}{2}}\left\|f^{0}\right\|_{\dot{H}_{x}^{1-\alpha / 2}}+\left\|f^{0}\right\|_{\dot{H}_{x}^{1 / 2}}^{\alpha}\|h\|_{\dot{H}_{x}^{1}}^{1-\alpha}+\varepsilon^{1-\alpha / 2}\|g\|_{\dot{H}_{x}^{1-\alpha / 2}}\right. \\
& \left.+\|g\|_{L^{2}}^{\alpha / 2}\|h\|_{\dot{H}_{x}^{1}}^{1-\alpha / 2}+\|h\|_{\dot{H}_{x}^{1}}^{1-\alpha / 2}\left\|\frac{h-f}{\varepsilon}\right\|_{L^{2}}^{\alpha / 2}\right)
\end{aligned}
$$

4. Relaxation limit. This section is devoted to the proof of the a priori estimates that enable to apply compensated compactness to the approximate solutions $\rho_{\varepsilon}, u_{\varepsilon}$ obtained in Section 2 .

4.1. Estimate in $H^{1}$. We shall assume that vacuum does not appear. Since $\rho_{\varepsilon} \in L_{x}^{1}$, we cannot have a global lower bound, thus we assume a local lower bound,

$$
\rho_{\varepsilon}(t, x) \geq \eta_{T, R} \quad \text { for } 0 \leq t \leq T,-R<x<R,
$$

for some $\eta_{T, R}>0$ independent of $\varepsilon$. 
THEOREM 4.1. We assume that $\lambda \geq 1$, that is to say $1<\gamma \leq 5 / 3$, and that the solution $f_{\varepsilon}$ to (2.1)-(2.10) with initial data $f_{\varepsilon}^{0} \in H_{x}^{1 / 2}\left(\mathbb{R}_{x} \times \mathbb{R}_{\xi}\right.$ ) such that (2.22)-(2.24) hold and

$$
\left\|f_{\varepsilon}^{0}\right\|_{H_{x}^{1 / 2}\left(\mathbb{R}_{x} \times \mathbb{R}_{\xi}\right)} \leq \frac{C}{\varepsilon^{1 / 2}},
$$

satisfies (2.35)-(2.36) and (4.1). We set $U_{\varepsilon}=\left(\rho_{\varepsilon}, \rho_{\varepsilon} u_{\varepsilon}\right)=\int f_{\varepsilon} d \xi$. Then, there exists $C_{T, R}>0$, independent of $\varepsilon$, such that for any $0<\varepsilon \leq \varepsilon_{0}$,

$$
\begin{array}{r}
\iint_{] 0, T[\times]-R, R[}\left|\partial_{x} U_{\varepsilon}\right|^{2} d t d x \leq \frac{C_{T, R}}{\varepsilon}, \\
\iint_{] 0, T[\times]-R, R[}\left|\partial_{t} U_{\varepsilon}\right|^{2} d t d x \leq \frac{C_{T, R}}{\varepsilon} .
\end{array}
$$

If moreover $f_{\varepsilon}^{0} \in H_{x}^{1}\left(\mathbb{R}_{x} \times \mathbb{R}_{\xi}\right)$ with

$$
\left\|f_{\varepsilon}^{0}\right\|_{H_{x}^{1}\left(\mathbb{R}_{x} \times \mathbb{R}_{\xi}\right)} \leq \frac{C}{\varepsilon}
$$

then

$$
\iiint_{[0, T[\times]-R, R[\times \mathbb{R}}\left|\partial_{x} f_{\varepsilon}\right|^{2} d t d x d \xi+\iiint_{[0, T[\times]-R, R[\times \mathbb{R}}\left|\partial_{t} f_{\varepsilon}\right|^{2} d t d x d \xi \leq \frac{C_{T, R}}{\varepsilon} .
$$

Proof. Obviously, we can apply Proposition 3.1 to a vector function. We take $a(\xi)=\xi$ and $\psi \in C_{c}(\mathbb{R}), 0 \leq \psi \leq 1$, such that $\psi=1$ on the support in $\xi$ of $f_{\varepsilon}$. Then (3.1) holds with $\alpha=1, K=1$, and we have $\rho_{\psi}=\int f_{\varepsilon} \psi(\xi) d \xi=U_{\varepsilon}$. Therefore we obtain with $h=M\left[f_{\varepsilon}\right]$ and $g=0$

$$
\left\|U_{\varepsilon}\right\|_{\dot{H}_{x}^{1 / 2}(] 0, T[\times \mathbb{R})} \leq C\left[\left\|f_{\varepsilon}^{0}\right\|_{L_{x, \xi}^{2}}+\left\|M\left[f_{\varepsilon}\right]\right\|_{L_{t, x, \xi}^{2}}^{1 / 2}\left\|\frac{M\left[f_{\varepsilon}\right]-f_{\varepsilon}}{\varepsilon}\right\|_{L_{t, x, \xi}^{2}}^{1 / 2}\right] .
$$

According to Theorem 2.5, we conclude that

$$
\left\|U_{\varepsilon}\right\|_{\dot{H}_{x}^{1 / 2}(] 0, T[\times \mathbb{R})} \leq C_{T} \varepsilon^{-1 / 4} .
$$

Then, we estimate $M\left[f_{\varepsilon}\right]=M\left(U_{\varepsilon}, \xi\right)$. We localize in $x$ by taking a function $\zeta_{R}(x) \in$ $C_{c}^{\infty}, \zeta_{R} \geq 0$, such that $\zeta_{R}(x)=1$ for $-R \leq x \leq R$. Since on the support of $\zeta_{R}, \rho_{\varepsilon}$ is lower bounded by (4.1), we have that there $M(., \xi)$ is Lipschitz continuous, because $\lambda \geq 1$. Therefore, we deduce that

$$
\left\|\zeta_{R}(x) M\left[f_{\varepsilon}\right]\right\|_{H_{x}^{1 / 2}(] 0, T\left[\times \mathbb{R}_{x} \times \mathbb{R}_{\xi}\right)} \leq C_{T, R} \varepsilon^{-1 / 4} .
$$

We then write the characteristics formula

$$
f_{\varepsilon}(t, x, \xi)=f_{\varepsilon}^{0}(x-t \xi, \xi) e^{-t / \varepsilon}+\frac{1}{\varepsilon} \int_{0}^{t} e^{-s / \varepsilon} M\left[f_{\varepsilon}\right](t-s, x-s \xi, \xi) d s .
$$


By multiplying by $\zeta_{R}(x)$ and by taking the $H_{x}^{1 / 2}$ norm, we obtain by using the translation invariance that for fixed $t, \xi$

$$
\begin{aligned}
& \left\|\zeta_{R}(x) f_{\varepsilon}(t, x, \xi)\right\|_{H_{x}^{1 / 2}\left(\mathbb{R}_{x}\right)} \\
\leq & \left\|\zeta_{R}(x+t \xi) f_{\varepsilon}^{0}(x, \xi)\right\|_{H_{x}^{1 / 2}\left(\mathbb{R}_{x}\right)} e^{-t / \varepsilon} \\
& +\frac{1}{\varepsilon} \int_{0}^{t} e^{-s / \varepsilon}\left\|\zeta_{R}(x+s \xi) M\left[f_{\varepsilon}\right](t-s, x, \xi)\right\|_{H_{x}^{1 / 2}\left(\mathbb{R}_{x}\right)} d s .
\end{aligned}
$$

Since $\xi$ remains in a bounded domain, we conclude with (4.9) that

$$
\left\|\zeta_{R}(x) f_{\varepsilon}\right\|_{H_{x}^{1 / 2}(] 0, T\left[\times \mathbb{R}_{x} \times \mathbb{R}_{\xi}\right)} \leq C_{T, R} \varepsilon^{-1 / 4} .
$$

Next, we would like to use the estimate of Corollary 3.2, but we need to localize in $x$. We write

$$
\partial_{t}\left(\zeta_{R} f_{\varepsilon}\right)+\xi \partial_{x}\left(\zeta_{R} f_{\varepsilon}\right)=\frac{\zeta_{R} M_{\varepsilon}-\zeta_{R} f_{\varepsilon}}{\varepsilon}+\xi \zeta_{R}^{\prime} f_{\varepsilon},
$$

thus we can apply Corollary 3.2 to $f_{\varepsilon}^{R}=\zeta_{R} f_{\varepsilon}$ with $h=\zeta_{R} M_{\varepsilon}$ and $g=\xi \zeta_{R}^{\prime} f_{\varepsilon}$. We obtain

$$
\begin{aligned}
& \quad\left\|\partial_{x}\left(\zeta_{R} U_{\varepsilon}\right)\right\|_{L_{t, x}^{2}} \\
& \leq C\left[\left\|\zeta_{R} f_{\varepsilon}^{0}\right\|_{\dot{H}_{x}^{1 / 2}\left(\mathbb{R}_{x} \times \mathbb{R}_{\xi}\right)}+\varepsilon^{1 / 2}\left\|\xi \zeta_{R}^{\prime} f_{\varepsilon}\right\|_{\dot{H}_{x}^{1 / 2}(] 0, T\left[\times \mathbb{R}_{x} \times \mathbb{R}_{\xi}\right)}\right. \\
& \left.\quad+\left\|\partial_{x}\left(\zeta_{R} M_{\varepsilon}\right)\right\|_{L_{t x \xi}^{2}}^{1 / 2}\left(\left\|\xi \zeta_{R}^{\prime} f_{\varepsilon}\right\|_{L_{t x \xi}^{2}}^{1 / 2}+\left\|\zeta_{R} \frac{M_{\varepsilon}-f_{\varepsilon}}{\varepsilon}\right\|_{L_{t x \xi}^{2}}^{1 / 2}\right)\right] .
\end{aligned}
$$

We notice that since $M\left[f_{\varepsilon}\right]=M\left(U_{\varepsilon}, \xi\right)$, we have $\partial_{x} M\left[f_{\varepsilon}\right]=M^{\prime}\left(U_{\varepsilon}, \xi\right) \partial_{x} U_{\varepsilon}$. One can check that $\rho_{\varepsilon}, u_{\varepsilon}$ being bounded, we have

$$
\left\|M^{\prime}\left(U_{\varepsilon}, \xi\right)\right\|_{L_{\xi}^{2}} \leq C \rho_{\varepsilon}^{-\frac{3}{4}(\gamma-1)}
$$

this is true indeed for $1<\gamma<2$, and this is a bit more general than the Lipschitz argument used in (4.9). We deduce with (4.14), (4.12) and Theorem 2.5 that

$$
\left\|\zeta_{R} \partial_{x} U_{\varepsilon}\right\|_{L_{t, x}^{2}} \leq C_{T, R}\left[\varepsilon^{-1 / 2}+\varepsilon^{-1 / 4}\left\|\zeta_{R} \partial_{x} U_{\varepsilon}\right\|_{L_{t, x}^{2}}^{1 / 2}\right],
$$

which gives (4.3). Then, we multiply (4.13) by $\xi$ and apply again Corollary 3.2, which gives that $\left\|\zeta_{R} \partial_{x} \int \xi f_{\varepsilon} d \xi\right\|_{L_{t x}^{2}} \leq C_{T, R} \varepsilon^{-1 / 2}$, and since $\partial_{t} U_{\varepsilon}+\partial_{x} \int \xi f_{\varepsilon} d \xi=0$, we get (4.4). The last estimate (4.6) is finally obtained by replacing the $H^{1 / 2}$ norm by the $H^{1}$ norm in (4.11), and by expressing $\partial_{t} f_{\varepsilon}$ from the BGK equation with the estimate of Theorem 2.5.

4.2. Entropy equations and compensated compactness. We conclude here the proof of Theorem 1.1, by establishing the strong convergence of $U_{\varepsilon}$. It is obtained by the method of DiPerna [12].

Proof of Theorem 1.1. We have from (2.29)

$$
\partial_{t} U_{\varepsilon}+\partial_{x} \int_{\mathbb{R}} \xi f_{\varepsilon} d \xi=0,
$$


thus by $(1.5)$

$$
\partial_{t} U_{\varepsilon}+\partial_{x} F\left(U_{\varepsilon}\right)=W_{\varepsilon}, \quad \text { with } W_{\varepsilon}=\partial_{x} \int_{\mathbb{R}} \xi\left(M\left[f_{\varepsilon}\right]-f_{\varepsilon}\right) d \xi .
$$

Take now a smooth entropy $\eta$, with entropy flux $G$. Recalling that by Theorem 4.1, $U_{\varepsilon} \in H_{t x}^{1}$ locally, we multiply (4.18) by $\eta^{\prime}\left(U_{\varepsilon}\right)$, and get

$$
\partial_{t} \eta\left(U_{\varepsilon}\right)+\partial_{x} G\left(U_{\varepsilon}\right)=\eta^{\prime}\left(U_{\varepsilon}\right) \cdot W_{\varepsilon} .
$$

Now

$$
\begin{aligned}
& \eta^{\prime}\left(U_{\varepsilon}\right) \cdot W_{\varepsilon} \\
= & \partial_{x}\left[\eta^{\prime}\left(U_{\varepsilon}\right) \cdot \int_{\mathbb{R}} \xi\left(M\left[f_{\varepsilon}\right]-f_{\varepsilon}\right) d \xi\right]-\partial_{x}\left[\eta^{\prime}\left(U_{\varepsilon}\right)\right] \cdot \int_{\mathbb{R}} \xi\left(M\left[f_{\varepsilon}\right]-f_{\varepsilon}\right) d \xi,
\end{aligned}
$$

therefore we get

$$
\partial_{t} \eta\left(\rho_{\varepsilon}, u_{\varepsilon}\right)+\partial_{x} G\left(\rho_{\varepsilon}, u_{\varepsilon}\right)=m_{\eta, \varepsilon}+R_{\eta, \varepsilon}
$$

with

$$
m_{\eta, \varepsilon}=-\eta^{\prime \prime}\left(U_{\varepsilon}\right) \cdot \partial_{x} U_{\varepsilon} \cdot \int_{\mathbb{R}} \xi\left(M\left[f_{\varepsilon}\right]-f_{\varepsilon}\right) d \xi
$$

and

$$
R_{\eta, \varepsilon}=\partial_{x}\left[\eta^{\prime}\left(U_{\varepsilon}\right) \cdot \int_{\mathbb{R}} \xi\left(M\left[f_{\varepsilon}\right]-f_{\varepsilon}\right) d \xi\right] .
$$

Using Theorem 2.5, we obtain

$$
\eta^{\prime}\left(U_{\varepsilon}\right) \cdot \int_{\mathbb{R}} \xi\left(M\left[f_{\varepsilon}\right]-f_{\varepsilon}\right) d \xi \rightarrow 0 \quad \text { in } L_{t, x}^{2}, \quad \text { as } \varepsilon \rightarrow 0,
$$

and indeed also in $L^{p}$ for $p<\infty$ since this is bounded in $L^{\infty}$. Furthermore, by Theorems 4.1 and 2.5,

$$
\begin{aligned}
& \iint_{] 0, T[\times]-R, R[}\left|\partial_{x} U_{\varepsilon}\right| \cdot\left|\int_{\mathbb{R}} \xi\left(M\left[f_{\varepsilon}\right]-f_{\varepsilon}\right) d \xi\right| d t d x \\
& \leq C\left\|\partial_{x} U_{\varepsilon}\right\|_{L^{2}(] 0, T[\times]-R, R[)}\left\|M\left[f_{\varepsilon}\right]-f_{\varepsilon}\right\|_{L_{t, x, \xi}^{2}} \leq C_{T, R} .
\end{aligned}
$$

which yields that $m_{\eta, \varepsilon}$ is bounded in $\mathcal{M}_{l o c}$ uniformly with respect to $\varepsilon$. We obtain therefore that $\partial_{t} \eta\left(\rho_{\varepsilon}, u_{\varepsilon}\right)+\partial_{x} G\left(\rho_{\varepsilon}, u_{\varepsilon}\right)$ is compact in $H_{l o c}^{-1}$. We are now able to apply compensated compactness, and the result of [20] allows to conclude the compactness of the sequence $\left(\rho_{\varepsilon}, \rho_{\varepsilon} u_{\varepsilon}\right)$. By passing to the limit in (4.18), we obtain (1.2) together with the initial data. Finally, it remains to establish an inequality for the physical entropy. We use a differential form of (2.30), that is fully justified in [3],

$$
\partial_{t} \int H\left(f_{\varepsilon}, \xi\right) d \xi+\partial_{x} \int \xi H\left(f_{\varepsilon}, \xi\right) d \xi \leq 0
$$

which gives with $(2.17),(2.18)$ that

$$
\begin{aligned}
& \partial_{t} \eta^{e}\left(\rho_{\varepsilon}, u_{\varepsilon}\right)+\partial_{x} G^{e}\left(\rho_{\varepsilon}, u_{\varepsilon}\right) \\
\leq & \partial_{t} \int\left(H\left(M\left[f_{\varepsilon}\right], \xi\right)-H\left(f_{\varepsilon}, \xi\right)\right) d \xi+\partial_{x} \int \xi\left(H\left(M\left[f_{\varepsilon}\right], \xi\right)-H\left(f_{\varepsilon}, \xi\right)\right) d \xi \\
& \longrightarrow 0 \text { in } W_{l o c}^{-1, p},
\end{aligned}
$$


and the desired result.

REMARK. The above proof shows that the rate $\left\|M\left[f_{\varepsilon}\right]-f_{\varepsilon}\right\|_{L^{2}} \leq C \varepsilon^{1 / 2}$ cannot be improved, otherwise we would get in the relaxation limit a solution that satisfies entropy equalities, which is not possible in general. The rates of Theorem 4.1 are therefore also sharp, since they say that all the terms of the BGK equation are of the same order in terms of $\varepsilon$. However, for other norms than $L^{2}$, the rate could be improved. For example, one can have $\left\|M\left[f_{\varepsilon}\right]-f_{\varepsilon}\right\|_{L^{1}} \leq C \varepsilon$, this should be somehow related to a BV bound.

Acknowledgement. This research was supported, for the first author, by the French-Austrian "Amadeus" program (ÖAD V6) and by the Austrian START project (TEC-Y137) "Nonlinear Schrödinger and quantum Boltzmann equations".

\section{REFERENCES}

[1] C. Bardos, F. Golse, C.-D. Levermore, The acoustic limit for the Boltzmann equation, Arch. for Ration. Mech. and Anal., 153 (2000), pp. 177-204.

[2] F. Berthelin, F. Bouchut, Solution with finite energy to a BGK system relaxing to isentropic gas dynamics, Annales de la Faculté des Sciences de Toulouse, 9 (2000), pp. 605-630.

[3] F. Berthelin, F. Bouchut, Kinetic invariant domains and relaxation limit from a BGK model to isentropic gas dynamics, Asymptotic Analysis, 31 (2002), pp. 153-176.

[4] F. Berthelin, F. Bouchut, Weak entropy boundary conditions for isentropic gas dynamics via kinetic relaxation, J. Diff. Eq., 185 (2002), pp. 251-270.

[5] F. Berthelin, F. Bouchut, Weak solutions for a hyperbolic system with unilateral constraint and mass loss, to appear in Ann. IHP Analyse non linéaire (2003).

[6] F. Bouchut, Construction of BGK models with a family of kinetic entropies for a given system of conservation laws, J. Statist. Phys., 95 (1999), pp. 113-170.

[7] F. Bouchut, Renormalized solutions to the Vlasov equation with coefficients of bounded variation, Arch. for Ration. Mech. and Anal., 157 (2001), pp. 75-90.

[8] F. Bouchut, Hypoelliptic regularity in kinetic equations, to appear in J. Math. Pures Appl. (2002).

[9] F. Bouchut, L. Desvillettes, Averaging lemmas without time Fourier transform and application to discretized kinetic equations, Proceedings of the Royal Society of Edinburgh, 129A (1999), pp. 19-36.

[10] F. Bouchut, F. Golse, M. Pulvirenti, Kinetic equations and asymptotic theory, Series in Appl. Math., Gauthiers-Villars, 2000.

[11] G.Q. Chen, C.D. Levermore, T.-P. Liu, Hyperbolic conservation laws with stiff relaxation terms and entropy, Comm. Pure Appl. Math., 47 (1994), pp. 787-830.

[12] R.J. DiPerna, Convergence of approximate solutions to conservation laws, Arch. Rational Mech. Anal., 82 (1983), pp. 27-70.

[13] R.J. DiPerna, P.-L. Lions, Y. Meyer, $L^{p}$ regularity of velocity averages, Ann. Inst. H. Poincaré Anal. Non Linéaire, 8 (1991), pp. 271-287.

[14] F. Golse, P.-L. Lions, B. Perthame, R. Sentis, Regularity of the moments of the solution of a transport equation, J. Funct. Anal., 76 (1988), pp. 110-125.

[15] F. Golse, L. SAint-Raymond, The Navier-Stokes limit of the Boltzmann equation: convergence proof, preprint.

[16] S. HwANG, Kinetic decomposition for kinetic models of BGK type, preprint.

[17] S. Hwang, A.E. Tzavaras, Kinetic decomposition of approximate solutions to conservation laws: applications to relaxation and diffusion-dispersion approximations, Comm. Partial Diff. Eq., 27 (2002), pp. 1229-1254.

[18] P.-E. Jabin, B. Perthame, Regularity in kinetic formulations via averaging lemmas, preprint.

[19] P.-L. Lions, N. Masmoudi, From the Boltzmann equations to the equations of incompressible fluid mechanics I, II, Arch. Ration. Mech. Anal., 158 (2001), pp. 173-193, 195-211.

[20] P.-L. Lions, B. Perthame, P.E. Souganidis, Existence and stability of entropy solutions for the hyperbolic systems of isentropic gas dynamics in Eulerian and Lagrangian coordinates, Comm. Pure Appl. Math., 49 (1996), pp. 599-638.

[21] P.-L. Lions, B. Perthame, E. Tadmor, Kinetic formulation of the isentropic gas dynamics and p-systems, Comm. Math. Phys., 163 (1994), pp. 415-431. 
[22] B. Perthame, P.E. Souganidis, A limiting case for velocity averaging, Ann. Sci. École Norm. Sup., (4) 31 (1998), pp. 591-598.

[23] L. Saint-RAYmond, From the Boltzmann BGK equation to the Navier-Stokes system, preprint.

[24] D. Serre, Relaxation semi-linéaire et cinétique des systèmes de lois de conservation, Ann. Inst. H. Poincaré Anal. non linéaire, 17 (2000), pp. 169-192.

[25] L. TARTAR, Compensated compactness and applications to partial differential equations, Nonlinear analysis and mechanics: Heriot-Watt Symposium, Vol. IV, pp. 136-212, Res. Notes in Math. 39, Pitman, Boston, Mass.-London, 1979.

[26] A.E. Tzavaras, Materials with internal variables and relaxation to conservation laws, Arch. Ration. Mech. Anal., 146 (1999), pp. 129-155.

[27] A.E. Tzavaras, Derivation of fluid equations for kinetic models with one conserved quantity, preprint. 
F. BERTHELIN AND F. BOUCHUT 